\title{
Non-encapsulated thermo-liquid crystals for digital particle tracking thermography/velocimetry in microfluidics
}

\author{
Rodrigo Segura $\cdot$ Christian Cierpka $\cdot$ Massimiliano Rossi $\cdot$ \\ Sonja Joseph · Heike Bunjes · Christian J. Kähler
}

Received: 26 May 2012/Accepted: 31 August 2012/Published online: 23 October 2012

(C) The Author(s) 2012. This article is published with open access at Springerlink.com

\begin{abstract}
The ever accelerating state of technology has powered an increasing interest in heat transfer solutions and process engineering innovations in the microfluidics domain. In order to carry out such developments, reliable heat transfer diagnostic techniques are necessary. Thermo-liquid crystal (TLC) thermography, in combination with particle image velocimetry, has been a widely accepted and commonly used technique for the simultaneous measurement and characterization of temperature and velocity fields in macroscopic fluid flows for several decades. However, low seeding density, volume illumination, and low TLC particle image quality at high magnifications present unsurpassed challenges to its application to three-dimensional flows with microscopic dimensions. In this work, a measurement technique to evaluate the color response of individual non-encapsulated TLC particles is presented. A Shirasu porous glass membrane emulsification approach was used to produce the nonencapsulated TLC particles with a narrow size distribution and a multi-variable calibration procedure, making use of all three RGB and HSI color components, as well as the proper orthogonally decomposed RGB components, was used to achieve unprecedented low uncertainty levels in the temperature estimation of individual particles, opening the door to simultaneous temperature and velocity tracking using 3D velocimetry techniques.
\end{abstract}

R. Segura ( $\square)$ · C. Cierpka · M. Rossi · C. J. Kähler Institute of Fluid Mechanics and Aerodynamics,

Universität der Bundeswehr, 85577 Neubiberg, Germany

e-mail: rodrigo.segura@unibw.de

C. Cierpka

e-mail: christian.cierpka@unibw.de

S. Joseph $\cdot$ H. Bunjes

Institute of Pharmaceutical Technology, Technische Universität

Braunschweig, 38106 Braunschweig, Germany
Keywords Microfluidics · Individual · Thermo-liquid crystal · TLC · Thermography · Non-encapsulated · Multi-variable calibration

\section{Introduction}

Heat transfer problems are commonplace in modern science and engineering and their solutions have led to a long list of technological developments, many of which have played a major role in transportation, communications, consumer electronics and personal computing, amongst many other areas that affect our lives and society. Today, with the strong downward trend in the size of electronic components down to micro and nano scales, combined with the upward trend in their heat tolerance demand, innovations in heat transfer solutions become more and more relevant. Experimental techniques used to solve heat transfer problems have played a substantial role in understanding a myriad of the physical phenomena that occur in its many applications. One common way to estimate temperatures by means of optical methods is the use of laser induced fluorescence (LIF) or luminescence. The physical principle is that the fluorescence intensity of a dye dissolved in the fluid changes as a function of temperature. In order to decrease the uncertainty due to illumination variations, ratiometric LIF uses the signals of two different dyes, where only one exhibits a temperature dependence. In microfluidics, however, flow fields are most often fully three-dimensional and in many instances include strong gradients. Hence, a temperature measurement approach that does not integrate across the flow volume, such as the tracking of individual particles, is preferable. Someya et al. (2011) used a high speed camera to measure the 2D velocity and temperature fields using particles, labeled with a temperature dependent luminophore, and 
evaluating their luminescence's lifetime. Only recently, Vogt and Stephan (2012) showed results of an experiment where particles were labeled with two dyes. They reported higher uncertainties compared to dissolved dyes but proved that the technique works using averages over interrogation windows. Nevertheless, two-dye particle labeling is not currently a common procedure, thus making the particles very expensive. Another way to determine the temperature is to evaluate the Brownian motion of particles (Hohreiter et al. 2002). The major drawback of this method is that in order to be able to reliably measure Brownian motion, the particles have to be very small, thus substantially decreasing their signal. Furthermore, their size distribution must be very narrow and the viscosity of the fluid should not change if a consistent relationship between Brownian motion and temperature is to be established. Furthermore, only time averaged measurements are possible. Besides these techniques, liquid-crystal thermography has received great attention over the last several decades due to its capability to quickly reconstruct temperature fields in surfaces and flow planes/ volumes. Thermochromic liquid crystals (TLCs) are substances whose molecular structure has both solid and liquid properties, hence the name liquid crystal, that change their color as a function of temperature (Fergason 1966; Adams et al. 1969; Parsley 1991).

An extensive amount of research has been done on the subject and the latest comprehensive review articles covering a wide range of aspects regarding this technique are provided by Dabiri (2009) and Abdullah et al. (2010). They thoroughly report and discuss most of the available literature, dating back to the $1800 \mathrm{~s}$, on several subjects that concern this technique such as the motivations for its development, properties of TLCs, colorimetry, imaging, commercial availability, time response, calibration, uncertainty analysis, hysteresis, and applications, among others. Research has been done using TLCs in thin films that are applied to surfaces to measure their temperature (Ireland and Jones 1987; Akino et al. 1989; Farina 1995; Sabatino et al. 2000) and the concept has even been extended to the microscopic domain with the aim of measuring twodimensional temperature fields or point-wise average heat transfer coefficients in the walls of microtubes and channels (Höhmann and Stephan 2002; Muwanga and Hassan 2006a, b, 2007; Chin et al. 2002). The principle of the technique has also been combined with particle image velocimetry (PIV) to reconstruct the temperature of flow fields (Dabiri and Gharib 1991) and simultaneously measure their velocity distribution in two dimensions. This task has been accomplished by several researchers over the last two decades, carefully tabulated and outlined in Table 1 of Dabiri's (2009) review and Tables 2.1 and 2.2 of Nasarek's (2010) report, and the technique continues to evolve into better and more efficient temperature and velocity measurement methods. It is worth noting that in order to simultaneously measure the flow temperature and velocity, researchers have seeded the flow with solutions containing TLC material as well as tracer particles, only suited for cross-correlation velocimetry, due to the low image quality of the individual TLC particles. Up to this day, all of the reports on this flow diagnostics technique are based on acquiring color images of flows, with high concentrations of TLC material, and reconstructing planar temperature fields. Extensions of this principle like scanning methods (Fujisawa and Funatani 2000; Fujisawa et al. 2005; Lutjen et al. 2001) and stereoscopic arrangements (Funatani and Fujisawa 2002; Fujisawa et al. 2004, 2005) have also been used to reconstruct three-dimensional temperature flow fields. All of these measurements rely on the concept of breaking the color images into sections and evaluating the mean temperature of the flow in each section. Even though sophisticated methods have been successfully employed to extract the mean temperature of the flow by evaluating these so-called interrogation windows (Park et al. 2001), the resolution of such measurements is limited by the size of the windows and the reconstruction of temperature gradients is necessarily biased when using this approach. Furthermore, when the flow volumes are reduced to microscopic dimensions, the measurement paradigm changes and, for one, the use of a light sheet is not possible thus introducing another bias in the measurement of flows that contain a temperature gradient in the depth direction. Secondary flows, commonly encountered in microchannel geometries, possess flow features that are completely hidden to two-dimensional measurements with volume illumination (Rossi et al. 2011). On the other hand, as explained in Cierpka and Kähler (2012)'s review, seeding concentration tends to be lower in microfluidics experiments and correlation methods become less reliable while the individual tracking of particles would be a convenient solution to avoid bias errors. Moreover, if the TLC particles have a narrow size distribution, astigmatism particle tracking velocimetry (APTV) can be used to track their motion in three dimensions as well as their temperature (Cierpka et al. 2010, 2011). To this day, however, the application of TLC thermography, combined with particle velocimetry, to three-dimensional microflows presents currently unsurpassed challenges caused by sparse seeding density, volume illumination, and low particle image quality at high magnifications. Even though Park et al. (2001) also used the average temperature over sections to reconstruct the flow's two-dimensional temperature field, they reported uncertainty figures for the temperature estimation of individual particles ranging from around 5 to $20 \%$ over a temperature span of $2.5 \mathrm{~K}$ using a neural network calibration approach. They used micro-encapsulated TLCs manufactured by Hallcrest (BM40C26W20) 
with a useful temperature response range approximately between 26 and $29^{\circ} \mathrm{C}$. Due to the high uncertainty in the temperature measurement of individual particles, they calculated the mean temperature over interrogation windows of $32 \times 32$ pixel and obtained lower errors of $2-8 \%$. This reduction in uncertainty, however, comes at the expense of a lower spatial resolution due to the averaging over interrogation windows. Basson and Pottebaum (2012), recently reported temperature measurements in a microchannel using individual encapsulated TLC particles. They demonstrate interesting advantages provided by an innovative circularly polarized illumination system on micro-encapsulated TLCs also manufactured by Hallcrest (NSL33R35C5 W), yielding average uncertainties of $1.2 \mathrm{~K}$ over a temperature range of 36.3 and $43.7{ }^{\circ} \mathrm{C}(16 \%)$.

Over the years, many calibration methods have been engineered to extract the temperature from the color of TLCs. Section 5 of Dabiri (2009)'s review and Section 5.3 of Abdullah et al. (2010)'s report provide a thorough account of the various attempts to perform this task over the last two decades. Rao and Zang (2010), Bednarz et al. (2010), and Cukurel et al. (2012) are some of the latest reports of novel techniques and ideas on this topic that are not covered in the earlier reviews. The general tendency over the last decades has been to use the temperature-hue relationship of TLCs to calibrate the digital images, where hue is a parameter that represents a color shade and will be defined in more detail in Sect. 4 On the other hand, multivariable calibration approaches using the color images' hue $(\mathrm{H})$, saturation (S), and intensity (I) have also been studied, since the $S$ and I signals also contain valuable information that plays a substantial role in relating the TLCs' temperature to the color of their digital images. Fujisawa and Hashizume (2001) performed a study of synthetical data, using a multi-variable calibration approach, and reported uncertainty values of $0.06 \mathrm{~K}$ in a range of $2.3 \mathrm{~K}(2.6 \%)$ for two-dimensional interrogation windows. Fujisawa et al. (2005) later reported uncertainty values of $0.13 \mathrm{~K}$, using a stereoscopic setup, on TLCs with a temperature response range of $2.3 \mathrm{~K}(5.7 \%)$, using a similar approach. It should be noted, however, that even though the HSI color space has been the transformation of choice for most research groups using this measurement technique, it is not the only means of interpreting color of digital images in order to extract their temperature information. Roesgen and Totaro (2002) introduced a statistical calibration approach that uses proper orthogonal decomposition (POD) to transform the raw RGB digital image data into a fully decorrelated variable space with the potential of providing a steeper calibration curve that yields a lower uncertainty for a oneor two-variable calibration. Hence, a study of non-encapsulated micro-TLC particles is presented to track the temperature of individual tracers in microfluidic flows using a multi-variable calibration approach. The tracer particles were fabricated by Shirasu Porous Glass (SPG) membrane emulsification, a technique that allows for the production of particles with narrow size distributions (Joscelyne and Trägårdh 2000).

\section{Experimental setup}

All experiments were performed at the microfluidics laboratory of the Bundeswehr University Munich (UniBw). The imaging system used to capture the flow snapshots is schematically shown in Fig. 1 and consists of an Axio Observer Z.1 inverted microscope manufactured by Carl Zeiss AG, a HXP $120 \mathrm{~W}$ flash lamp, and a JAI AT-200 GE, 3CCD Progressive Scan RGB Color, 24-bit compounded dynamic range (8-bit per sensor), 1,624 × 1,236 pixel $^{2}$ digital color camera. The sensor alignment of the color camera was evaluated and yielded a spatial discrepancies of less than 0.5 pixel.

The entire system was installed on a damped optical table to avoid the effect of vibrations in the measurements. The light was routed to the back aperture of the microscope via an optical fiber and relayed to the objective lens through a filter, mounted inside the microscope. An LD Plan-NEOFLUAR, $40 \times$, infinity corrected objective lens, with a numerical aperture of $\mathrm{NA}=0.6$, was used to focus the light on the flow sample, as well as to image the flow itself. A droplet of TLC-containing emulsion (see Sect. 3) was poured on a microscope glass plate and a Peltier element was mounted immediately above the droplet such that the fluid was in direct contact with most of its heating surface. The thickness of the fluid layer (TLC droplet) between the glass plate and the surface of the Peltier element was approximately $1.5 \mathrm{~mm}$. The temperature of the Peltier element was automatically controlled by a custom module, implemented in the in-house image acquisition software, written in MATLAB, via a Peltier-Controller TC2812 manufactured by CoolTronic GmbH and a mini Pt1000 thermocouple. The acquisition frame rate for all

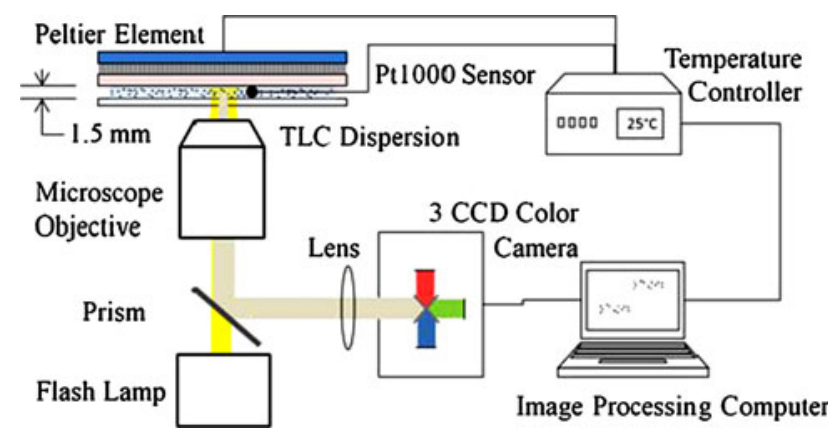

Fig. 1 Schematic of experimental setup 
measurements was $15 \mathrm{fps}$ and a field of view of $400 \times 300 \mu \mathrm{m}^{2}$ was used for the temperature response analysis. The nuclei of the particle images used for the calibration analysis were approximately 20 pixel in diameter, on average. The TLC containing droplet remained in contact with the peltier element for approximately one hour, which was the time required for the temperature scanning procedure, and no abrasion or bleeding was observed in the TLC particles. The sample was only illuminated for the short lapses of time required for image acquisition (the microscope shutter was opened once the desired temperature was reached and the camera was ready to acquire, and subsequently closed after images were recorded) and no bleaching was observed in the TLC material under these conditions. Nevertheless, a preliminary bleaching test was performed to assess the resistance of the TLC and a degradation of the particles' color was observed after approximately $30 \mathrm{~min}$ of continuous illumination. The time response of the TLC material was not measured for this study. The reader can refer to Ireland and Jones (1987) for a study on the response time of TLC thin sheets, where they report delay times of $3 \mathrm{~ms}$.

\section{Dispersion of non-encapsulated TLC particles}

A major factor behind the high uncertainty of temperature measurements in individual TLC particles, such as those reported by Park et al. (2001), is the fact that the polymer shell around the TLC material causes aberrations and distorts the detected color of each particle creating a substantial bias error in the evaluation of color, especially for high magnification images. Additionally, the encapsulation process may also alter the molecular structure of the TLC material causing each particle to react differently to temperature changes. Figure 2 shows an example of what a particle from a sample of Hallcrest encapsulated TLCs (NSL40/R25C5W) looks like over its useful temperature range. Even though the color variation with temperature is clearly visible, the particle image displays a very strong outer ring that occurs due to the strong reflection of light from the encapsulating material, which produces errors that affect the evaluation process. Furthermore, complex structures inside the particle are evident, which vary from particle to particle. A brief study was performed using a solution of water and micro-encapsulated TLCs to track the temperature of individual particles using a Hue calibration as was done by Park et al. (2001) and Segura et al. (2011). The study yielded uncertainty values of $5 \%$ for the mean of particle images, which are comparable to those reported by Park et al. (2001) for the mean of $32 \times 32$ pixel $^{2}$ interrogation windows, and $16.6 \%$ for individual particles, which are also on the order of those reported by Park et al. (2001) for their individual encapsulated TLCs. This is why the successful temperature tracking of individual TLC particles requires several improvements to the current state-of-the-art TLC thermography technique. First of all, the quality of the particle images must be considerably better than that of encapsulated TLC particles. Second, a preprocessing algorithm that works consistently for the varying color intensity profiles that correspond to different temperatures is necessary to reliably detect all particles in the temperature range. And third, a reliable relationship between the color of each particle and its temperature must be established.

Since encapsulated TLC particles are clearly not a viable option for individual temperature tracking, nonencapsulated material was used. Thermography using non-encapsulated TLC material has been investigated to a limited extent for two-dimensional or point-wise temperature measurements (Muwanga and Hassan 2006a, b, 2007) but no attempts to fabricate stable TLC droplets for particle tracking have been made so far. The TLC particles used in this study were produced at the Institute of Pharmaceutical Technology of the Braunschweig University of Technology using a direct SPG membrane emulsification process as described in detail by Nakashima et al. (1992). The TLC bulk material (Hallcrest UN R25C10W) was briefly heated to $55^{\circ} \mathrm{C}$, beyond its melting point of $45-47^{\circ} \mathrm{C}$, and placed in a $10 \mathrm{ml}$ custom built, double walled, temperature controlled, pressurized, stainless steel, external pressure microkit-type vessel manufactured by MCTech Co. The device was equipped with a Haake D1 water bath, manufactured by Thermo Haake $\mathrm{GmbH}$, used for tempering the lipid phase of the material. A pressure of $25-35 \mathrm{kPa}$ was applied to force the lipid phase through a pre-wetted hydrophilic SPG membrane with a pore size of $5.5 \mu \mathrm{m}$, fabricated by SPG technology Co., into $80 \mathrm{ml}$ of $5 \%$ poloxamer 188 emulsifier (aqueous phase) with $0.01 \%$ thiomersal as preservative. The solution was continually stirred with a temperature controlled magnetic stirrer (IKAWerke $\mathrm{GmbH}$ ) at $300 \mathrm{rpm}$. Evaporated water was replenished every $12 \mathrm{~h}$ during the $48 \mathrm{~h}$ of preparation. After the direct emulsification process, the solution container was dipped into ice-water for at least $20 \mathrm{~min}$, giving birth to the TLC particles that would be used for the temperature tracking experiments, which take form when the emulsion droplets are cooled below the liquid crystal phase transition temperature. All samples were stored at room temperature $\left(18-20{ }^{\circ} \mathrm{C}\right)$. A direct comparison of the resulting nonencapsulated particles' raw color appearance with their encapsulated counterpart is displayed in Fig. 2. The nonencapsulated TLC particles display a much more homogeneous color profile and, even though the raw images' contrast seems to be low at higher temperatures, the color profile of the particle image stands out enough for the detection algorithm, outlined in detail in Sect. 4. 
Encapsulated

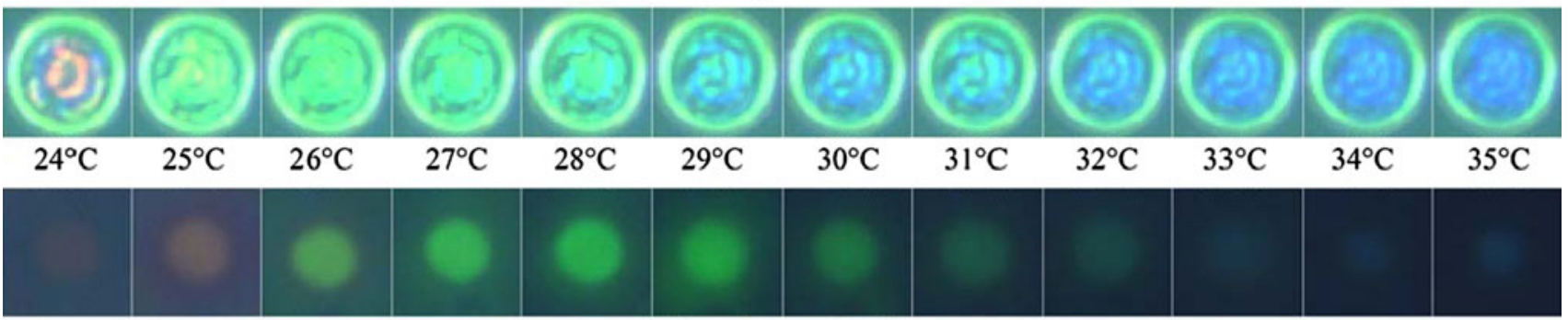

Non-Encapsulated

Fig. 2 Top: Raw color appearance of encapsulated TLC particles from Hallcrest Inc. at different temperatures $(20 \times$ magnification). Bottom: Raw color appearance of custom made non-encapsulated TLC particles at different temperatures (40× magnification)

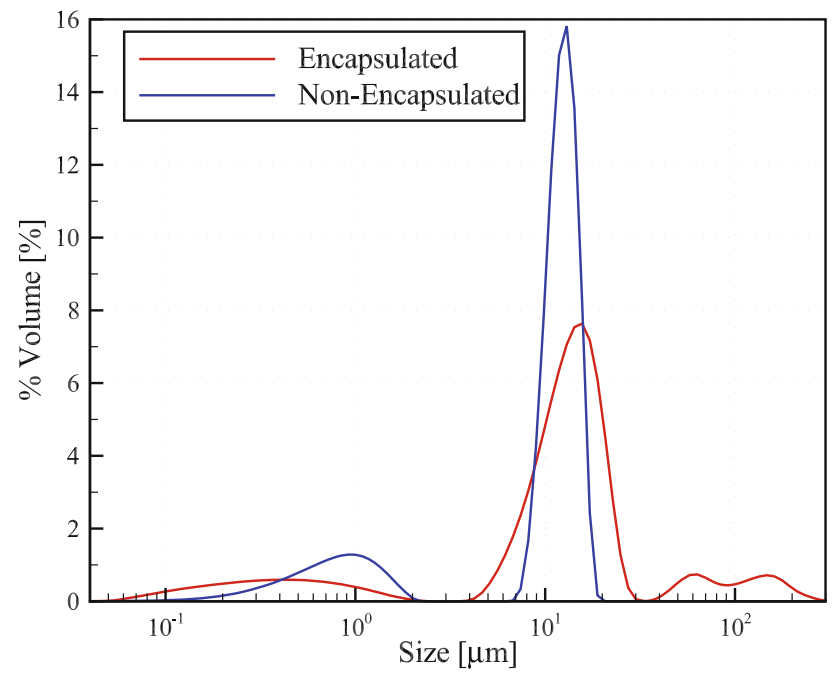

Fig. 3 Size distribution of custom made non-encapsulated TLC particles along with commercially available encapsulated TLC particles from Hallcrest

A laser diffractometer with polarization intensity differential tecnology (model LS 12320 by Beckman-Coulter) was used to estimate the particle size distribution of the dispersion. Three measurements were performed to calculate the volume distributions assuming an index of refraction of 1.46 for the TLC particles, 1.33 for the aqueous dispersion medium, and 0.01 for the imaginary part of the refractive index. The estimated particle size distribution for the nonencapsulated TLC particles is plotted in Fig. 3 along with that of micro-encapsulated TLC particles from Hallcrest (NSL40/R25C5W). Both sets display multi-modal distributions but the dominant diameter $(\sim 13 \mu \mathrm{m})$ of the nonencapsulated particles represents a higher relative volume, with a narrower distribution than that of their larger encapsulated counterpart $(\sim 16 \mu \mathrm{m})$. Furthermore, no higher diameter particles were detected in the non-encapsulated sample and the $\sim 1 \mu \mathrm{m}$ portion is not discretely discernable with the optical system used for the temperature tracking experiments.

\section{Image processing}

The digital color images consist of three matrices of data provided by three separate CCD sensors in the digital camera. Each one of these sensors is exposed to a different range of wavelengths and acquires the 'red' (R), 'green' $(\mathrm{G})$, and 'blue' (B) digital information of the image to produce a so-called 'RGB' color image (Fig. 4a). As the particles change their color, their R, G, B, intensity profiles change in a non-linear fashion. This makes the particle detection process more complex than with gray scale images such as those used for most PIV/PTV experiments. Since a real experiment would comprise flow regions of different temperatures, and thus particles of multiple colors, a method that can detect particles with different color profiles is necessary. Several approaches were tested and the following method worked consistently throughout the temperature range over which the particles display a color response (25-35 K). First, a weighted sum of the R, G, B components of the RGB image was calculated to generate a gray scale intensity image (Fig. 4b) that would serve as a basis for the image preprocessing and particle detection algorithms. Next, vertical and horizontal gradient filters with a 3 pixel kernel were applied in order to calculate the gradient magnitude over each kernel and detect particle image edges (Fig. 4c). This was done, instead of using other commonly used methods such as intensity thresholds, histogram filters, Gaussian filters, etc., because the particles' gray scale profiles vary substantially for different colors, and neither of these performed consistently well over the span of the evaluated temperature range. An automatic segmentation threshold was then calculated over the gradient magnitude map, to obtain a binary image, maximizing the separability of its black and white values as described by Otsu (1979) (Fig. 4d). This generates a black and white image of the rings surrounding the particles, which was subject to another filter used to fill all the closed perimeters, producing a black and white picture of sort-of swollen particle images (Fig. 4e). These segmented 
particle images, however, include most of the color gradient from their center to the background so an erosion filter was applied to reduce their size while preserving their general geometry (Fig. 4f). The reason for this preprocessing step is that the average intensity/color values of the particle image may be of use at a later time and including the gradient was found to strongly detriment the measurement results. Park et al. (2001) calculated the average over the adjacent pixels to the center of the particles, which should work, in principle, for circular particle images. However, if APTV were to be used to track these particles in a 3D volume (Cierpka et al. 2010, 2011), preserving the geometry of the elliptical particle images before calculating the average intensity/color values could improve the measurement outcome. Now, this binary image of the particle nuclei was used as a mask to calculate the exact positions of the particle images, in this case simply done by finding the centroid of the segmented nuclei pixel area (Fig. 4g, h), and to filter outlier particles' images by shape, size, perimeter and area before analyzing their color properties. Figure 5 shows a plot of the R, G, and B intensities at the center of all particles detected in the 10 images that were acquired at each temperature. The pronounced trends in the individual R, G, and B data profiles are evident from the figure and have been used by other research groups to directly calibrate the temperature response of TLC thin sheets in surface coatings (Vejrazka and Marty 2007). However, these values are all directly affected by the light-source's intensity and orientation, digital camera settings, optical arrangements, etc., and do not necessarily respond to variations of these experimental factors in the same manner, thus making them very susceptible to bias errors in the temperature calibration.

An alternative to directly using the R, G, and B components of color is to use the $\mathrm{H}, \mathrm{S}$, and I (HSI) color space. This is an alternative perspective of color perception that uses relationships between R, G, and B instead of their raw values.

- $\mathrm{H}$ describes the shade of a particular color; conventional color names like yellow, orange, etc. are examples of hue.

- S quantifies the degree of purity of the color, also described as the extent to which the color is diluted with white.

- I relates to the chromatic-independent brightness of the color.

There have been several formulations to the transformation from RGB to HSI color space (Russ 2002; Hay and Hollingsworth 1996, 1998; Pratt 2007). For the purposes of this study, a simplified version of Hay and Hollingsworth (1996)'s formulation will be used, employed by the 'rgb2hsv' function of the MATLAB software package where,
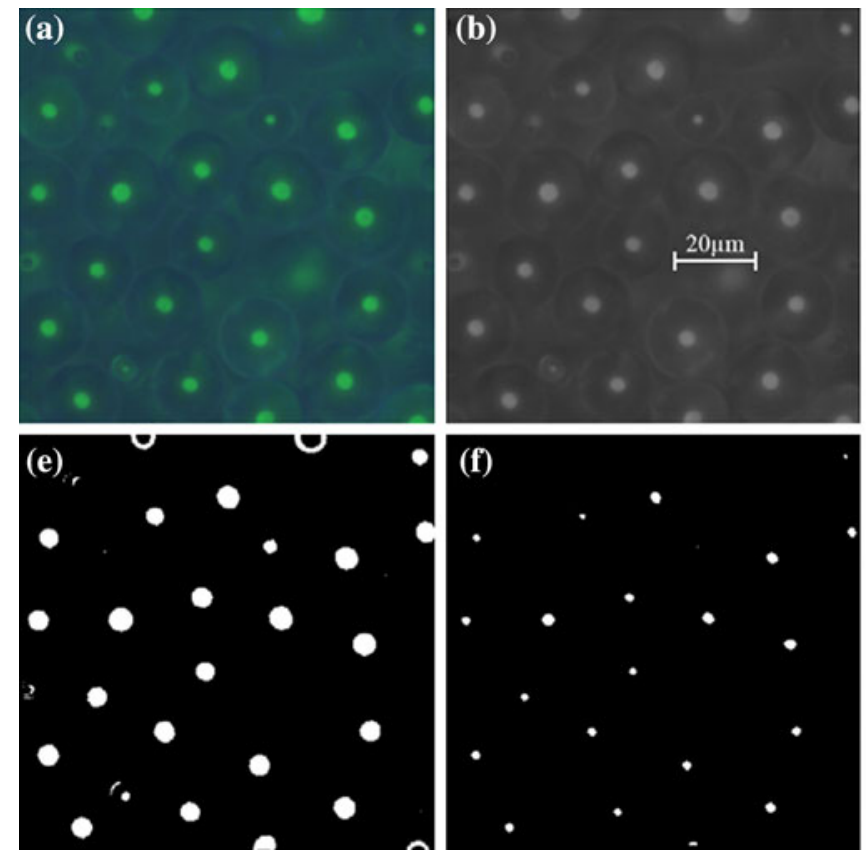

Fig. 4 a RGB image. b Gray scale image. c Gradient magnitude map over a 3 pixel kernel. d Black and white segmented particle image edges. e Filled particle image edges. $\mathbf{f}$ Reduced particle image centers
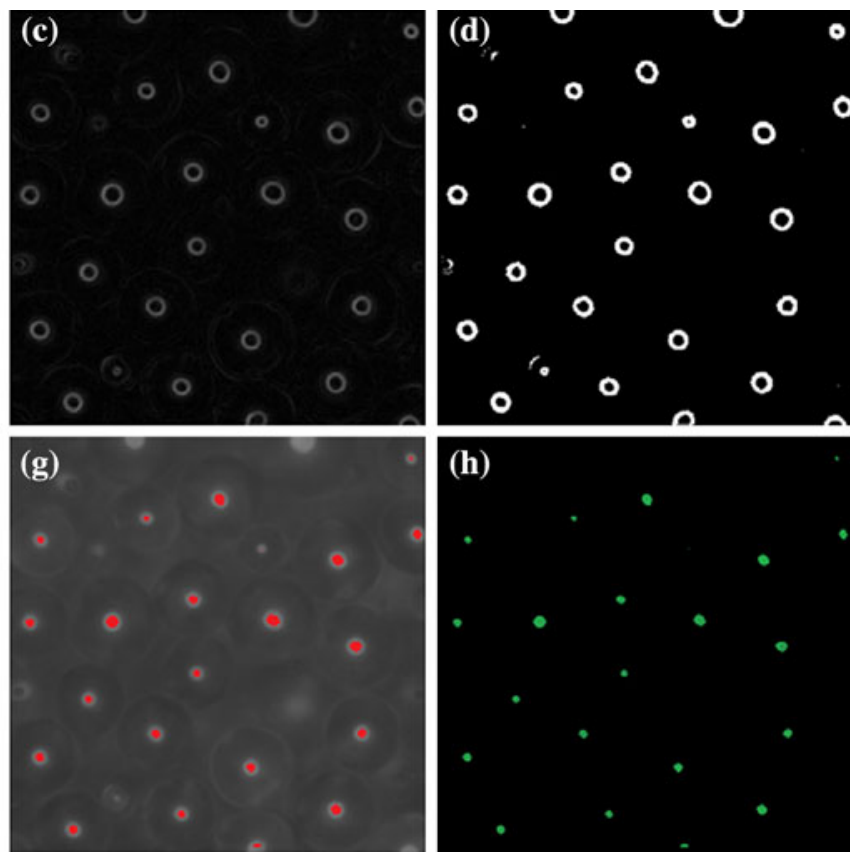

via an erosion filter. $\mathbf{g}$ Detected particle centers superimposed with the original gray scale image. $\mathbf{h}$ Isolated raw RGB centers of detected particle images 


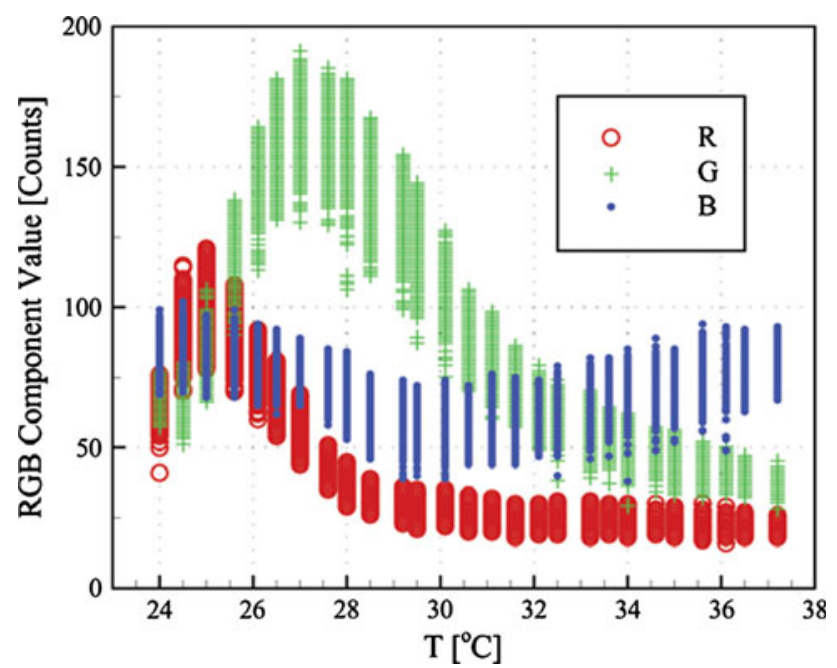

Fig. 5 Scatter plot of RGB values for all particles detected

$$
\begin{aligned}
I & =\max (R, G, B) \\
S & =I-\min (R, G, B) / I \\
H & =\frac{1}{6}\left\{\begin{array}{cc}
(G-B) I / S & \mid R>B, G \\
2+(B-R) I / S & \mid G>R, B \\
4+(R-G) I / S & \mid B>R, G
\end{array}\right\}
\end{aligned}
$$

Figure 6 shows a plot of the H, S, and I profiles of all the particles detected, over the temperature range evaluated. This transformation provides non-dimensonal HSI values, where $\mathrm{H}$ is a simplified approximation of Hay and Hollingsworth (1996) formulation, normalized with $2 \pi$, and $I$ is normalized with the maximum pixel count for an individual sensor of the digital camera (255 for each 8-bit sensor). Furthermore, the minimum and maximum hue and saturation values, respectively, detected in temperatures under the red start temperature of the TLC material $\left(25^{\circ} \mathrm{C}\right)$ were used as a threshold level under which the negative radian value of $\mathrm{H}$ would be used in order to obtain a better fit to a continuous third degree polynomial function.

The reader should note that the HSI color space is a nonreversible mathematical transformation of the raw images' RGB data. In the introduction of this transformation approach, Joblove and Greenberg (1978) states that most viewers notice a color's hue before they notice other characteristics so this convention arose as a convenient system for the specification of colors based on more humanly intuitive quantities than red, green and blue levels. Nevertheless, it is not the only way to interpret color and a more precise transformation may exist that is better suited for temperature estimation from the color of TLCs. Roesgen and Totaro (2002), for instance, introduced a statistical calibration approach that uses proper orthogonal decomposition (POD) to transform the raw RGB digital

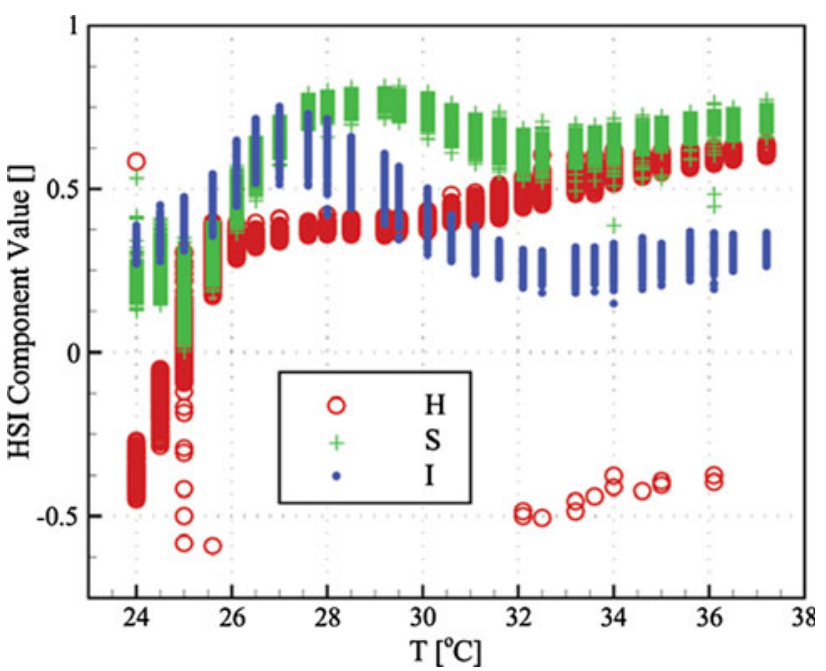

Fig. 6 Scatter plot of HSI values for all particles detected

image data into a fully decorrelated variable space with the potential of providing a steeper calibration curve that yields a lower uncertainty. They used normalized RGB values

$r=R /(R+G+B)$
$g=G /(R+G+B)$
$b=B /(R+G+B)$

to calculate the covariance matrix

$$
\begin{aligned}
C & =\operatorname{Cov}(r, g, b) \\
& =\left(\begin{array}{lll}
\overline{(r-\bar{r})^{2}} & \overline{(r-\bar{r})(g-\bar{g})} & \overline{(r-\bar{r})(b-\bar{b})} \\
\overline{(r-\bar{r})(g-\bar{g})} & \frac{(g-\bar{g})^{2}}{(g-\bar{g})(b-\bar{b})} \\
\frac{(r-\bar{r})(b-\bar{b})}{(g-\bar{g})(b-\bar{b})} & \frac{\overline{(b-\bar{b})^{2}}}{(g-\bar{b}}
\end{array}\right)
\end{aligned}
$$

where $\bar{x}=\frac{1}{n} \sum_{i=1}^{n} x_{i}$. The POD is then a linear transformation expressed as,

$$
\left(\begin{array}{l}
x_{1} \\
x_{2} \\
x_{3}
\end{array}\right)=\underline{\underline{T}}\left(\begin{array}{c}
(r-\bar{r}) \\
(g-\bar{g}) \\
(b-\bar{b})
\end{array}\right)
$$

where $\underline{T}$ is a rotation matrix such that $\underline{T}^{-1}=\underline{T}^{t}$, composed of the eigenvectors, arranged in rows below each other, of the eigenvalue problem

$C \vec{t}=\lambda \vec{t}$

The $x_{1}$ and $x_{2}$ components, associated with the direction of the non-zero eigenvalues $\lambda_{1}=0.0182$ and $\lambda_{2}=0.0075$ and plotted in Fig. 7, carry all the color information while the third component, associated with the direction of the lowest eigenvalue $\lambda_{3}=0$, vanishes due to the raw data normalization $(r+g+b=1)$. 


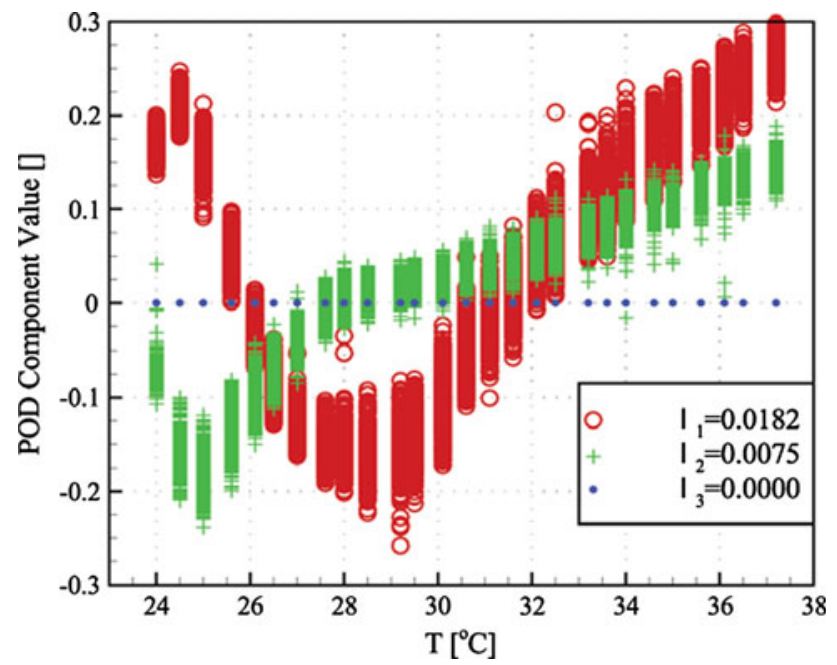

Fig. 7 Scatter plot of the POD components associated with the direction of the eigenvalues $\lambda_{1}=0.0182, \lambda_{2}=0.0075$ and $\lambda_{3}=0$ for all particles detected

\section{Calibration}

Just like with most measurement techniques, converting measured data to a desired physical quantity, in this case temperature, has been a topic of itself since the birth of the technique. So far, the hue-temperature dependence has been reported on most experimental studies as the basis for the calibration of the digital images even though a limited amount of research has been performed on directly using the $\mathrm{R}, \mathrm{G}, \mathrm{B}$ color components of digital images to extract the temperature (Vejrazka and Marty 2007). A thorough review of the literature pertinent to this particular topic can be found in Section 5.1 of Dabiri (2009)'s review and Section 5.3.1 of Abdullah et al. (2010)'s report. Rao and Zang (2010), Bednarz et al. (2010), and Cukurel et al. (2012) report the most recent studies and advances in the hue-temperature calibration of TLCs. Though this approach has worked for years and sophisticated computational methods such as neural networks have been used to improve the calibration results, it is clear from Fig. 6 that not only $\mathrm{H}$ shows a pronounced variation for different temperatures but $\mathrm{S}$ and $\mathrm{I}$ do as well. Now, if the $\mathrm{H}$ values can be successfully calibrated to temperature, it is only natural to expect that two more variables showing clear patterns would provide valuable information to improve the accuracy of the calibration.

A thorough calibration analysis was performed to find a method that accurately extracts the temperature from the color images of TLC particles. Even though RGB values are strongly affected by optical arrangements, and do not necessarily respond to experimental conditions variations in the same manner, thus making them very susceptible to bias errors in the temperature estimation, a calibration was performed using the raw RGB color components of the particle images as did Vejrazka and Marty (2007). Figure 8 shows a 3D scatter plot of the RGB components of all the particles detected with the temperature of each point given by the color map. This figure illustrates how all three color components provide valuable information required to precisely extract the temperature of particles that lie in the flat regions of the individual component profiles from Fig. 5, thus providing a pronounced data pattern to which a $3 \mathrm{D}$ polynomial can be fitted in order to obtain a calibration curve that works throughout the temperature range. Vejrazka and Marty (2007) used a similar approach to evaluate average two-dimensional temperature fields of a macroscopic impinging jet flow and reported uncertainty values of $<0.3 \mathrm{~K}$ in a range of $8.5 \mathrm{~K}(3.5 \%)$.

As mentioned in Sect. 4, an alternative using the HSI color space is available. The HSI color space uses the relationship between $\mathrm{R}, \mathrm{G}$, and $\mathrm{B}$ instead of their raw values. Furthermore, the relationship between temperature and hue value has been the calibration method used by the great majority of research groups over the years. Analogous to Figs. 5, 9 shows a 3D scatter plot of the HSI components of all the particles detected with the temperature of each point given by the color map. Conversely, this figure also illustrates how the $\mathrm{S}$ and I data provide valuable information required to precisely extract the temperature of particles that lie in the flat regions of the $\mathrm{H}$ profile from Fig. 6, once again providing a pronounced data pattern to which a $3 \mathrm{D}$ polynomial can be fitted in order to obtain a calibration curve that works throughout the temperature range. Fujisawa and Hashizume (2001) performed a study with synthetical data and compared results for the calibration of temperature using its relationship with $\mathrm{H}, \mathrm{H}$ and $\mathrm{S}$, and $\mathrm{H}, \mathrm{S}$ and I. They state that there is a clear advantage to using the $\mathrm{S}$ and I image data, in addition to $\mathrm{H}$, and reported an uncertainty of $0.06 \mathrm{~K}$ in a range of $2.3 \mathrm{~K}(2.6 \%)$, evaluating the average temperature in interrogation windows. Fujisawa et al. (2005) later reported uncertainty values of $0.13 \mathrm{~K}$, using a stereoscopic setup (also 2D interrogation windows), on TLCs with a temperature response range of $2.3 \mathrm{~K}(5.7 \%)$, also calibrating temperature with all three variables. Note that all of the research done up to this point has been performed using mean color values, calculated over designated areas of the sensor, and not individually for each tracer particle. As mentioned in previous sections, this fact is of major relevance for experiments in microfluidics since the recorded images integrate the light over the entire illuminated volume, introducing a strong bias in two-dimensional averages that does not occur with three-dimensional particle tracking. The temperature of individual TLC microdroplets evaluated in this study was also calibrated using all three $\mathrm{H}, \mathrm{S}$ and I color variables evaluated at the center of each particle image. 

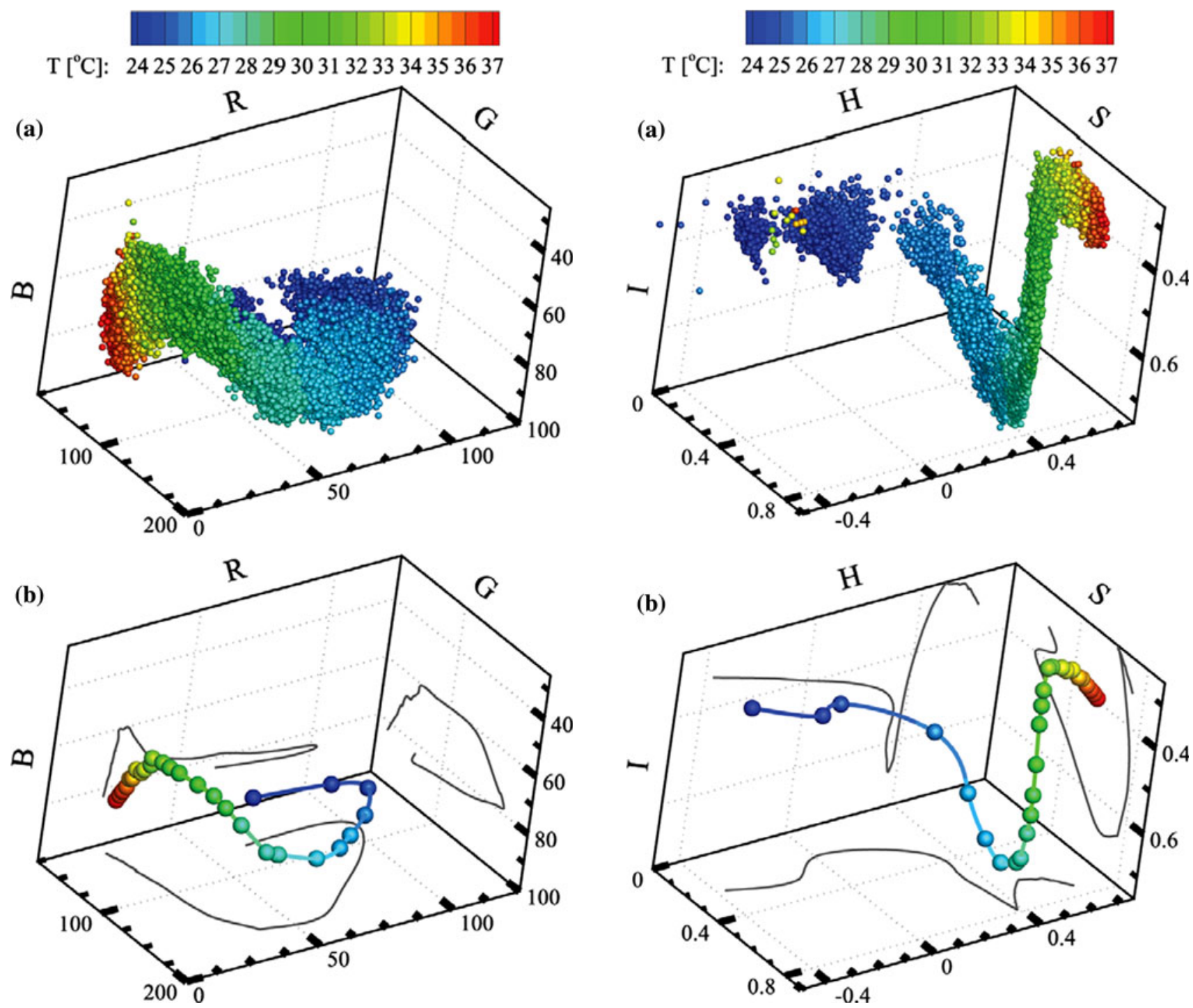

Fig. 8 a 3D scatter plot of RGB values for all particles detected. b Scatter plot of mean RGB values for detected particles at each temperature along with interpolated curve

A third variable space, resulting from the full decorrelation of the RGB image data via POD (Roesgen and Totaro 2002) as outlined in Sect. 4, was used to fit a 2D polynomial in order to obtain a calibration curve for the estimation of the individual particles' temperature. Figure 10 shows a 2D scatter plot of the $x_{1}$ and $x_{2}$ components, associated with the direction of the non-zero eigenvalues $\lambda_{1}=0.0182$ and $\lambda_{2}=0.0075$, of all the particles detected with the temperature of each point given by the color map.

Calibrations were made by fitting a three-dimensional third-degree polynomial to the RGB and HSI data and a two-dimensional third-degree polynomial to the POD data. This was done using a standard least-squares approach to solve the system of linear equations given by,

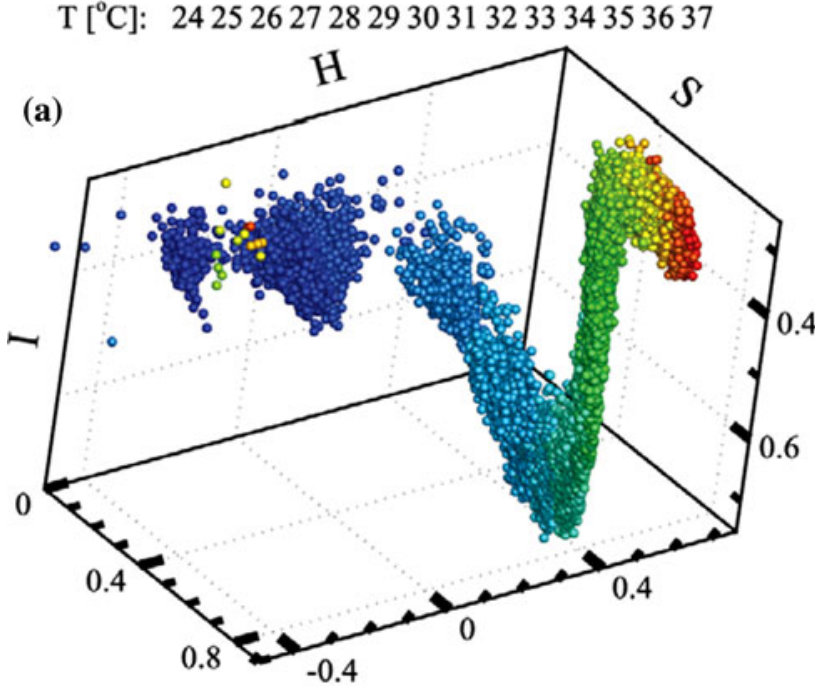

Fig. 9 a 3D scatter plot of HSI values for all particles detected. b Scatter plot of mean HSI values for detected particles at each temperature along with interpolated curve

$$
\left(\begin{array}{c}
T_{1} \\
T_{2} \\
\vdots \\
T_{i}
\end{array}\right)=\left(\begin{array}{c}
f\left(V_{1}, V_{1}, V_{2}\right)_{3} \\
f\left(V_{1}, V_{2}, V_{3}\right)_{2} \\
\vdots \\
f\left(V_{1}, V_{2}, V_{3}\right)_{i}
\end{array}\right) \cdot\left(\begin{array}{c}
c_{1} \\
c_{2} \\
\vdots \\
c_{k}
\end{array}\right)
$$

where $f\left(V_{1}, V_{2}, V_{3}\right)_{i}$ are arrays with the terms of the fit polynomial, corresponding to each particle's color space data, and $c_{k}$ are the corresponding fit coefficients.

\section{Results}

A stationary droplet of emulsion, containing the nonencapsulated TLC particles (see Sect. 3) was analyzed 


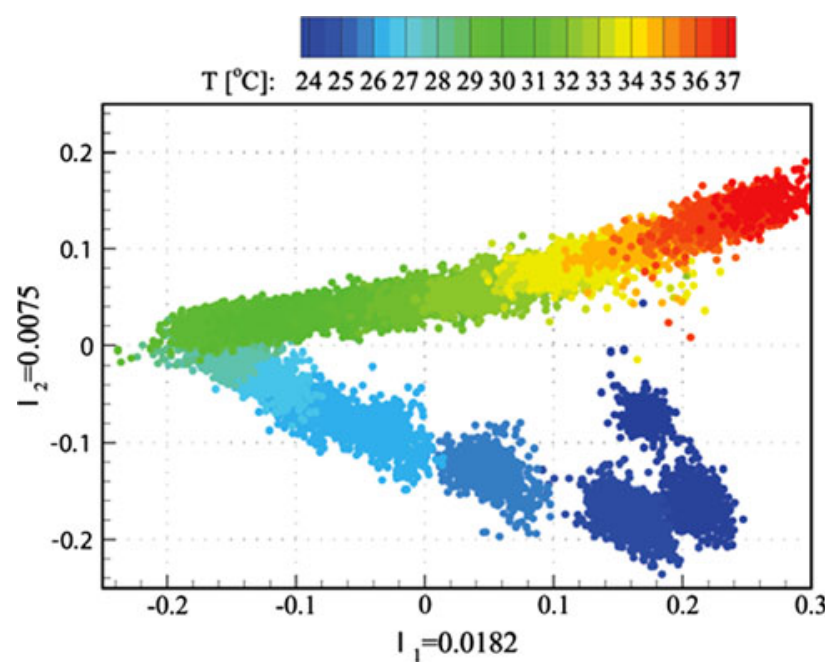

Fig. $102 \mathrm{D}$ scatter plot of the $x_{1}$ and $x_{2}$ components, associated with the direction of the non-zero eigenvalues $\lambda_{1}=0.0182$ and $\lambda_{2}=0.0075$ for all particles detected

between 22 and $38{ }^{\circ} \mathrm{C}$, across the nominal color response range of the TLC raw material quoted as $25-35{ }^{\circ} \mathrm{C}$ by Hallcrest Inc. Four images were analyzed at each $0.5 \mathrm{~K}$ step and the color response of the particle images was calibrated following the method outlined in Sect. 5 The number of detected particles per image was used as the criterion to set the limits to the useful temperature calibration range. An arbitrary threshold of 50 particles per image was used to define the range over which the temperature calibration would be evaluated, which resulted into a calibration of the image data corresponding to a temperature range of $24-37^{\circ} \mathrm{C}$. Note that this means that the image processing approach detects enough 'suitable for temperature calibration' particles over a temperature span beyond the nominal color response range of the TLC material. Figure 11 shows a plot of the $95 \%$ confidence interval uncertainty values for individual particles, as well as for the mean of all particles detected in each image, using the RGB, HSI, and POD variable space data. The $95 \%$ confidence interval uncertainty levels were calculated as two times the standard deviation of the difference between the estimated temperature and the actual one. All three variable spaces allow for the precise extraction of temperature. The calibration curve computed from the two POD variables exhibits higher uncertainties than the those obtained from using the three RGB and HSI variables, over the nominal temperature range of the TLC material $\left(25-35{ }^{\circ} \mathrm{C}\right)$. Furthermore, the uncertainty of the temperature estimation increases with the RGB calibration approach for temperatures beyond the nominal temperature response range of the TLC material $\left(35^{\circ} \mathrm{C}\right)$, surpassing the uncertainty values provided by the POD approach, and the HSI data yields uncertainties of $1-7 \%$ across a range of $25-37{ }^{\circ} \mathrm{C}$. Note that the multi-variable calibration of the three variables that result from applying POD to the raw RGB data (without normalization) yields exactly the same results as calibrating the raw RGB data directly. However, the calibration of temperature using hue alone, yields higher uncertainties than when the single $x_{1}$ POD component, associated with the direction of the largest eigenvalue $\lambda_{1}=0.0182$, is used. At any rate, the mean estimated temperatures using all RGB, HSI, and POD variable spaces exhibit lower uncertainties than the standard of $<3.5 \%$ reported by Vejrazka and Marty (2007). It is clear from the

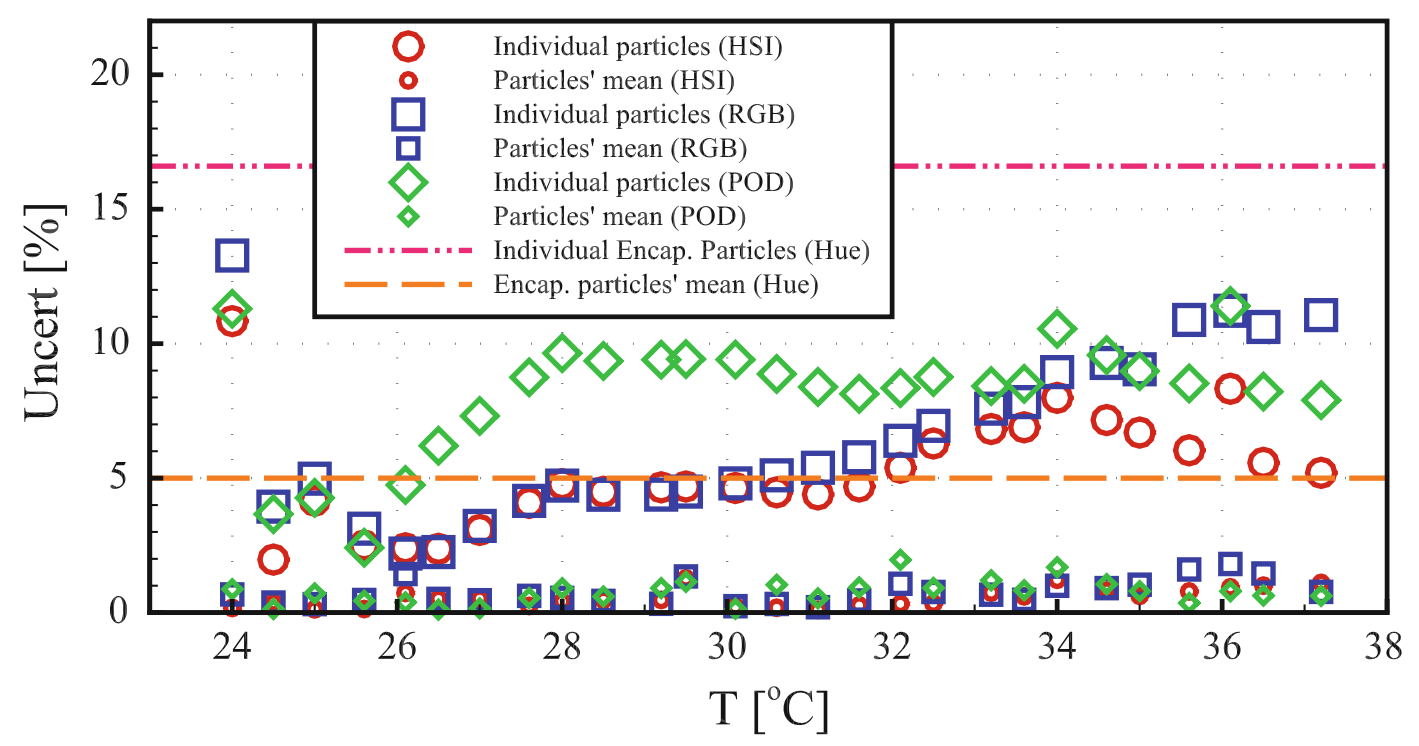

Fig. 11 Uncertainty values, with a $95 \%$ confidence interval, for individual non-encapsulated TLC particles as well as for the mean of all particles detected in each image using both the RGB and HSI color spaces. The horizontal dotted lines are uncertainty values, also with a $95 \%$ confidence interval, for encapsulated TLC particles from Hallcrest reported in Segura et al. (2011) 
figure that the calibration method works effectively on the individual particle image data, thus allowing for the precise evaluation of the flow temperature at discrete points in the volume. The reader should also note that the uncertainties reported above hold only for this particular experimental setup. Furthermore, this is the first method capable of tracking the temperature of individual flow tracers with these levels of uncertainty. The only other reports of temperature measurements in individual tracers were written by Park et al. (2001) and Basson and Pottebaum (2012) where they reported uncertainty values more than twice as high as those obtained in this study, though the confidence interval is unknown for Park et al. (2001) results. Park et al. (2001) used a sophisticated neural network calibration approach in highly seeded flow where particle tracking is rather challenging while Basson and Pottebaum (2012) experimented with an innovative circularly polarized illumination system, to reduce background noise. It is worth noting that the increasing trend of uncertainty with temperature for individual particles, observed in Fig. 11, does not seem to arise from a systematic mechanism that can be compensated for. It is rather a result of the increased proximity of the color component coordinates as the temperature increases, as can be observed in Figs. 8, 9.

\section{Conclusion}

A microscopically transparent emulsion, consisting of a dispersion of non-encapsulated TLC particles, was used to evaluate the performance of a multi-variable temperature calibration method to track individual tracers in a fluid volume. The non-encapsulated tracer particles were fabricated by Shirasu Porous Glass (SPG) membrane emulsification, yielding a narrower size distribution than that of their commercially available encapsulated counterparts, with the added advantage that they lack a polymer surface with a different index of refraction thus providing substantially improved particle images. The calibration uses the three components of the RGB and HSI color spaces and the two non-zero components of the POD variable space. A threedimensional polynomial was fitted to the color data of thousands of particles to estimate the temperature of each particle. The evaluation approach using all three HSI color components works consistently over a range exceeding the useful color response range of the TLC material, as quoted by the manufacturer, with an unprecedented low measurement uncertainty for individual temperature tracking of $4.9 \%$ over $13 \mathrm{~K}$, with a confidence interval of $95 \%$. This is a substantial step forward in the state-of-the-art of flow diagnostics concerning temperature measurements in microflows as the successful tracking of temperature in individual tracer particles opens the door to time resolved reconstruction of temperature flow fields. Furthermore, the combination of this measurement technique with astigmatism particle tracking velocimetry or other $3 \mathrm{D}$ defocusing methods has the potential of fully reconstructing time resolved velocity and temperature flow fields in threedimensions with high spatial resolution although challenges will arise due to lower signal-to-noise ratios in the defocused elliptical images of the TLC particles.

Acknowledgments Financial support from the German Research Foundation (DFG), under the framework of the mikroPART Collaborative Research Group (Micro systems for particulate Life-Science products), is gratefully appreciated.

Open Access This article is distributed under the terms of the Creative Commons Attribution License which permits any use, distribution, and reproduction in any medium, provided the original author(s) and the source are credited.

\section{References}

Abdullah N, Abu Talib AR, Jaafar AA, Mohd Salleh MA, Chong WT (2010) The basics and issues of thermochromic liquid crystal calibrations. Exp Thermal Fluid Sci 34: 1089-1121 doi: 10.1016/j.expthermflusci.2010.03.011

Adams JE, Haas W, Wysocki J (1969) Optical properties of certain cholesteric liquid-crystal films. J Chem Phys 50:2458-2464. doi: 10.1063/1.1671402

Akino N, Kunugi T, Ichimiya K, Mitsushiro K, Ueda M (1989) Improved liquid-crystal thermometry excluding human color sensation. J Heat Transf 111:558-565

Basson M, Pottebaum TS (2012) Measuring the temperature of fluid in a micro-channel using thermochromic liquid crystals. Exp Fluids. doi:10.1007/s00348-012-1326-0

Bednarz TP, Lei C, Patterson JC (2010) Various aspects of camera settings and image processing in the calibration of thermochromic liquid crystals for accurate particle image thermometry measurements. J Vis 13:241-250. doi:10.1007/s12650-0100038-x

Chin Y, Lakshminarasimhan MS, Lu Q, Hollingsworth DK, Witte LC (2002) Convective heat transfer in vertical asymmetrically heated narrow channels. J Heat Transf 124:1019-1025. doi: $10.1115 / 1.1497356$

Cierpka C, Kähler CJ (2012) Particle imaging techniques for volumetric three-component (3D3C) velocity measurements in microfluidics. J Vis 15:1-31. doi:10.1007/s12650-011-0107-9

Cierpka C, Segura R, Hain R, Kähler CJ (2010) A simple single camera $3 \mathrm{C} 3 \mathrm{D}$ velocity measurement technique without errors due to depth of correlation and spatial averaging for microfluidics. Meas Sci Technol 21:045401. doi:10.1088/0957-0233/21/4/ 045401

Cierpka C, Rossi M, Segura R, Kähler CJ (2011) On the calibration of astigmatism particle tracking velocimetry for microflows. Meas Sci Technol 22:015, 401. doi:10.1088/0957-0233/22/1/015401

Cukurel B, Selcan C, Arts T (2012) Color theroy perception of steady wide band liquid crystal thermometry. Exp Thermal Fluid Sci 39:112-122. doi:10.1016/j.expthermflusci.2012.01.015

Dabiri D (2009) Digital particle image thermometry/velocimetry: a review. Exp Fluids 46:191-241. doi:10.1007/s00348-008-0590-5

Dabiri D, Gharib M (1991) Digital particle image thermometry: the method and implementation. Exp Fluids 11:77-86 
Farina DJ (1995) Making surface temperature measurements using liquid crystal thermography. Electron Cool 1(2):10-15

Fergason JL (1966) Cholesteric structure-1 optical properties. Mol Cryst 1:293-307

Fujisawa N, Funatani S (2000) Simultaneous measurement of temperature and velocity in a turbulent thermal convetion by the extended range scanning liquid crystal visualization technique. Exp Fluids Suppl:S158-S165

Fujisawa N, Hashizume Y (2001) An uncertainty analysis of temperature and velocity measured by a liquid crystal visualization technique. Meas Sci Technol 12:1235-1242

Fujisawa N, Nakajima T, Katoh N, Hashizume Y (2004) An uncertainty analysis of temperature and velocity measured by stereo liquid-crystal thermometry and velocimetry. Meas Sci Technol 15:799-806. doi:10.1088/0957-0233/15/5/004

Fujisawa N, Funatani S, Katoh N (2005) Scanning liquid-crystal thermometry and stereo velocimetry for simultaneous threedimensional measurement of temperature and velocity field in a turbulent Rayleigh-Bernard convection. Exp Fluids 38:291-303. doi:10.1007/s00348-004-0891-2

Funatani S, Fujisawa N (2002) Simultaneous measurement of temperature and three velocity components in planar cross section by liquid-crystal thermometry combined with stereoscopic particle image velocimetry. Meas Sci Technol 13: 1187-1205

Hay JL, Hollingsworth DK (1996) A comparison of trichromic systems for use in the calibration of polymer-dispersed thermochromic liquid crystals. Exp Thermal Fluid Sci 12:1-12

Hay JL, Hollingsworth DK (1998) Calibration of micro-encapsulated liquid crystals using hue angle and a dimensionless temperature. Exp Thermal Fluid Sci 18:251-257

Höhmann C, Stephan P (2002) Microscalse temperature measurement at an evaporating liquid meniscus. Exp Thermal Fluid Sci 26: $157-162$

Hohreiter V, Wereley ST, Olsen MG, Chung JN (2002) Crosscorrelatioin analysis for temperature measurement. Meas Sci Technol 13:1072-1078

Ireland PT, Jones TV (1987) The response time of a surface thermometer employing encapsulated thermochromic liquid crystals. J Phys E Sci Instrum 20:1195-1199

Joblove GH, Greenberg D (1978) Color spaces for computer graphics. In: ACM SIGGRAPH 5th Annual Conference on Computer Graphics and Interactive Techniques, New York, USA, August

Joscelyne SM, Trägårdh G (2000) Membrane emulsification-a literature review. J Membr Sci 169:107-117

Lutjen PM, Mishra D, Prasad V (2001) Three-dimensional visualization and measurement of temperature field using liquid crystal scanning thermography. J Heat Transf 123:1006-1014. doi: $10.1115 / 1.1370514$

Muwanga R, Hassan I (2006a) Local heat transfer measurements in a curved microsurface using liquid crystal thermography. J Thermophys Heat Transf 20:884-894. doi:10.2514/1.17907

Muwanga R, Hassan I (2006b) Local heat transfer measurements in microchannels using liquid crystal thermography: methodology development and validation. J Heat Transf 128:617-626. doi: $10.1115 / 1.2193541$

Muwanga R, Hassan I (2007) A flow boiling heat transfer investigation of FC-72 in a microtube using liquid crystal thermography. J Heat Transf 129:977-987. doi:10.1115/1.2728905

Nakashima T, Shimizu M, Kukizaki M (1992) Membrane emulsification by microporous glass. Key Eng Mater 61-62:513-516. doi:10.4028/www.scientific.net/KEM.61-62.513

Nasarek R (2010) Temperature field measurement with high spatial and temporal resolution using liquid crystal thermography and laser induced fluorescence. $\mathrm{PhD}$ thesis, Technische Universität Darmstadt

Otsu N (1979) A thershold selection method from gray-level histograms. IEEE Trans Syst Man Cybernet 9:62-66

Park HG, Dabiri D, Gharib M (2001) Digital particle image velocimetry/thermometry and application to the wake of a heated circular cylinder. Exp Fluids 30:327-338. doi:10.1007/ s003480000199

Parsley M (1991) Handbook of thermochromic liquid crystal technology. Hallcrest, Glenview, IL

Pratt WK (2007) Digital image processing, 4th edn. Wiley, New York

Rao Y, Zang S (2010) Calibrations and the measurement uncertainty of wide-band liquid crystal thermography. Measur Sci Technol 21:015105. doi:10.1088/0957-0233/21/1/015105

Roesgen T, Totaro R (2002) A statistical calibration technique for thermochromic liquid crystals. Exp Fluids 33:732-734. doi: 10.1007/s00348-002-0525-5

Rossi M, Cierpka C, Segura R, Kähler CJ (2011) Volumetric reconstruction of the $3 \mathrm{D}$ boundary of stream tubes with general topology using tracer particles. Meas Sci Technol 22:105405. doi:10.1088/0957-0233/22/10/105405

Russ JC (2002) The Image Processing Handbook. CRC, Boca Raton

Sabatino DR, Praisner TJ, Smith CR (2000) A high-accuracy calibration technique for thermochromic liquid crystal temperature measurements. Exp Fluids 28:497-505

Segura R, Cierpka C, Rossi M, Kähler CJ (2011) Uncertainty analysis of particle image thermometry using individual thermo-liquid crystal tracers. In: 64th Annual Meeting of the APS Division of Fluid Dynamics, Baltimore, Maryland, USA, November 20-22

Someya S, Li Y, Ishii K, Okamoto K (2011) Combined twodimensional velocity and temperature measurements of natural convection using a high-speed camera and temperature-sensitive particles. Exp Fluids 50:65-73. doi:10.1007/s00348-010-0894-0

Vejrazka J, Marty P (2007) An alternative technique for the interpretation of temperature measurements using thermochromic liquid crystals. Heat Transf Eng 28:154-162. doi:10.1080/ 01457630601023641

Vogt J, Stephan P (2012) Using microencapsulated fluorescent dyes for simultaneous measurement of temperature and velocity fields. Measur Sci Technol 23:105306. doi:10.1088/0957-0233/ 23/10/105306 